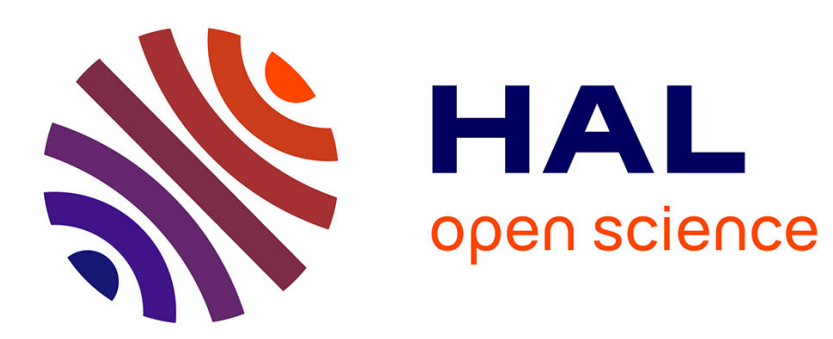

\title{
Comparison of two modulation techniques using frequency domain processing for in-house networks
}

Geert Carron, Reto Ness, Luc Deneire, L. van Der Perre, M.G.E. Engels

\section{To cite this version:}

Geert Carron, Reto Ness, Luc Deneire, L. van Der Perre, M.G.E. Engels. Comparison of two modulation techniques using frequency domain processing for in-house networks. IEEE Transactions on Consumer Electronics, 2001, 47 (1), pp.63-72. 10.1109/30.920421 . hal-00187735

\section{HAL Id: hal-00187735 https://hal.science/hal-00187735}

Submitted on 15 Nov 2007

HAL is a multi-disciplinary open access archive for the deposit and dissemination of scientific research documents, whether they are published or not. The documents may come from teaching and research institutions in France or abroad, or from public or private research centers.
L'archive ouverte pluridisciplinaire $\mathbf{H A L}$, est destinée au dépôt et à la diffusion de documents scientifiques de niveau recherche, publiés ou non, émanant des établissements d'enseignement et de recherche français ou étrangers, des laboratoires publics ou privés. 


\title{
COMPARISON OF TWO MODULATION TECHNIQUES USING FREQUENCY DOMAIN PROCESSING FOR IN-HOUSE NETWORKS
}

\author{
Geert Carron, Reto Ness, Luc Deneire, Liesbet Van der Perre and Marc Engels \\ \{carron, deneire, vdperre, engelsm\}@imec.be \\ Interuniversity Micro Electronics Center (IMEC) - Kapeldreef 75, 3001 Heverlee, Belgium
}

\begin{abstract}
Recently, the industry has shown a growing interest in the field of in-house wireless communication. The two major technical challenges are the interference and the signal distortion by the communication channel. We propose three different interference mitigation and distortion compensation strategies. These strategies are based on frequency domain processing, which is best supported by Orthogonal Frequency Division Multiplexing (OFDM) and Single-Carrier modulation with cyclic prefix (SCCP). Both modulation techniques are compared with respect to their performance in an in-house environment. Both techniques are promising for in-house communication in an environment corrupted by interference and frequency selective channels.
\end{abstract}

\section{Introduction}

Wireless digital communication in indoor environments is gaining interest of customers and industry. The performance of wireless indoor networks is however limited by the communication channel, which distorts the signal due to reflections and scattering. This channel distortion must be compensated such that a high data-throughput is reachable while keeping the system cost moderate. The compensation is performed by a channel equalizer. When the equalization is performed in the time domain, it requires a very complex block, such as a Viterbi equalizer. To avoid this complex block, we propose to do the equalization in the frequency domain.

Another way of reducing the system cost is using the $2.4 \mathrm{GHz}$ license-free Industrial, Scientific and Medical (ISM) band. However, as more systems make use of this ISM-band, an increasing level of mutual interference is generated. On top of this, the $2.4 \mathrm{GHz}$ band is corrupted by energy leakage of microwave ovens. A reliable communication system must also compensate this impairment.

This paper compares two systems that have interesting properties for wireless in-house communication: Orthogonal Frequency Division Multiplexing (OFDM) and Single Carrier modulation with cyclic prefix (SC-CP). OFDM is the modulation technique chosen in most of the current in-house communication standards. This modulation technique requires less digital complexity compared with the SC-CP technique, but has two major drawbacks : the OFDM-signal is characterized by a high peak-to-average and the system puts a high requirement on the frequency synchronization at the receiver. On top of the multipath-fading channel, two types of interference are used : the Bluetooth communication system and the microwave oven. Three interference mitigation techniques are introduced : adaptive loading and spreading with maximal-ratio combining for OFDM, and FRequency SHift (FRESH) filtering in the frequency-domain for SC-CP.

The results show that both systems are able to setup a successful high-speed communication in an in-house environment with static interference. Although OFDM requires less digital complexity, the OFDM signal properties require more analog complexity. Therefore, a trade-off between both systems must be made on a higher system-level including all the digital and analog aspects.

Section two introduces the channel model, the modulation techniques and compares their performance in a multipath channel. Section three derives the interference models while section four details the mitigation techniques and their performance.

\section{System Models}

In this section, we first introduce the channel model we used to evaluate the system performances. The following two parts present the two modulation techniques, OFDM and SC-CP. In a fourth part, we compare both techniques on a system level and present performance results for both systems.

\subsection{Channel Model}

A reliable model for the transmission channel is needed to analyze the performance of the different modulation techniques in an in-house environment. We have implemented a ray-tracing model[1] that generates the frequency- or time domain response of an indoor environment based on the geometrical layout of this environment. The ray-tracing program computes all paths from a transmitter to a receiver, taking the different reflections into account, until the total path attenuation has reached a predefined value. The length and the attenuation of the different paths is then used to compute the time- and frequency domain response. These 
ray-tracing simulations show us that the in-house channel is characterized by a RMS-delay spread $\tau_{R M S}$ of 5 to 20 ns. The frequency-domain channel responses show frequencydips of up to $30 \mathrm{~dB}$. We have used this model to generate 120 different in-house channel responses, which we used to perform the multipath performance simulations.

\subsection{The OFDM System}

The OFDM-system is a special case of multi-carrier transmission. In this kind of transmission systems, the signal bandwidth $W$ is subdivided in a number of sub-bands $N_{c}$. Each sub-band is used to transmit $1 / N_{c}$-th of the data. In a normal multi-carrier transmission system, the $N_{c}$ sub-bands are completely separated, such that no Inter-Carrier Interference (ICI) exists. In OFDM, ICI is avoided by using orthogonal subcarriers : $\int_{0}^{T} s_{i}(t) s_{j}(t) d t=\delta_{i j}$. At the receiver, we can reconstruct the data by sampling the received signal in the frequency-domain at the correct carrier frequencies. Figure 1(a) depicts the block diagram of an OFDM system. At the transmitter, complex constellation symbols, called datasymbols, are generated from the data. These symbols are used to modulate the different sub-carriers. The modulation and frequency-spacing is automatically performed by passing the complex constellation symbols through an Inverse Fast Fourier transform (IFFT). Afterwards, the outputsamples of the IFFT are multiplexed into an OFDM-symbol. A copy of the last $N_{g}$ samples of the OFDM symbol, called a cyclic-Prefix (CP), is prepended to each OFDM-symbol. This CP has two purposes : first it serves as a guard-interval between the different OFDM-symbols and secondly it transforms the OFDM-signal into a pseudo-cyclic signal. The guard-interval serves as a buffer between subsequent OFDMsymbols so that no InterSymbol Interference (ISI) is generated. All the echoes of one OFDM-symbol will fall in the $\mathrm{CP}$ of the next OFDM-symbol. At the receiver, the $\mathrm{CP}$ is removed and so is the ISI. The pseudo-cyclic signal has an important property that largely reduces the complexity of the receiver.

After the OFDM-symbol is multiplexed and a CP is prepended, the signal is transmitted over the indoor channel where noise and interference is added. At the receiver, the CP is removed and the OFDM-symbols are demultiplexed again. The sub-carriers are demodulated again into complex constellation points by applying a Fast Fourier Transform (FFT). This operation transforms the signal back to the frequency domain, were we perform the channel equalization, interference mitigation and data-detection. The channel-equalization effort is largely reduced by adding the $\mathrm{CP}$. An equalizerblock compensates for the channel distortion. This channeldistortion means that the transmitted signal is linearly convoluted with the time-domain impulse response of the channel. However, from digital signal processing theory ([2], page 415) we know that a cyclic convolution in the timedomain is transformed into a simple multiplication in the frequency-domain. By introducing the $\mathrm{CP}$, we have generated a pseudo-cyclic signal and thereby transformed the linear channel-convolution into a cyclic convolution. As a result, the complex time-domain equalizer is replaced by a simple multiplication in the frequency-domain for every subcarrier.

\subsection{The SC-CP System}

The single-carrier system with cyclic prefix depicted in Figure 1(b) aims to combine the advantages of the frequencydomain processing of OFDM, such as the reduced equalizer complexity, with the advantages of single-carrier transmission, such as the better peak-to-average ratio. The complexity of the equalizer is reduced by grouping the datasymbols into fixed-length block-symbols and insert a $\mathrm{CP}$ between these block-symbols. The CP avoids ISI and transforms the signal in a pseudo-cyclic signal. The block-symbols are multiplexed and transmitted over the channel. Noise and interference is added and the receiver demultiplexes the blocks again. Then, the $\mathrm{CP}$ is removed and the block-symbols are transformed to the frequency-domain to perform equalization and interference mitigation. Finally, the blocksymbols are retransformed to the time domain for data detection.

\subsection{System Comparison}

The two modulation techniques presented above have different signal properties. OFDM is in essence a multi-carrier modulation scheme, while SC-CP is a single-carrier modulation technique. This difference will have an implication on the performance of both systems and the requirements for the analog front-ends. This subsection discusses three important consequences.

A first consequence is the different behaviour of both systems in a multipath environment. This environment will selectively attenuate the signal on certain frequencies. For SC$\mathrm{CP}$, the selective attenuation results in a small energy loss for all transmitted data and thus a proportional reduced detection reliability. In the OFDM case, this selective attenuation will result in an almost complete information loss on certain subcarriers. This analysis suggests that an OFDM-system will perform worse than a single-carrier system, which is confirmed by the simulation results presented in Figure 2. This figure plots the Bit Error Rate (BER) with respect to $E_{b} / N_{0}$, which is the average bit energy divided by the double-sided spectral noise density. This difference disappears when we take Forward Error Coding and interleaving into account. By doing so, we introduce frequency diversity into the signal. This makes the OFDM-system equally performant as the single-carrier system.

A second consequence concerns the peak-to-average property of the transmitted signal. The signals on the different subcarriers in an OFDM-system have an uncorrelated phase. When all the subcarriers are in-phase, the OFDM-system 


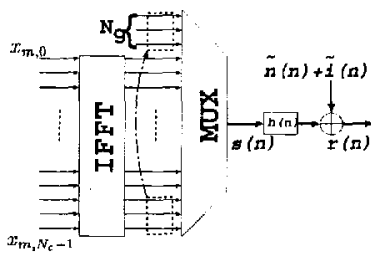

(a) OFDM

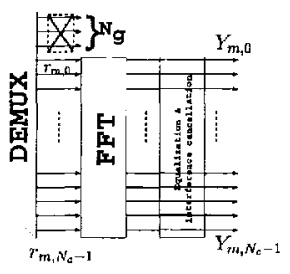

Figure 1: Single carrier transmission moves the processing to the receiver-part

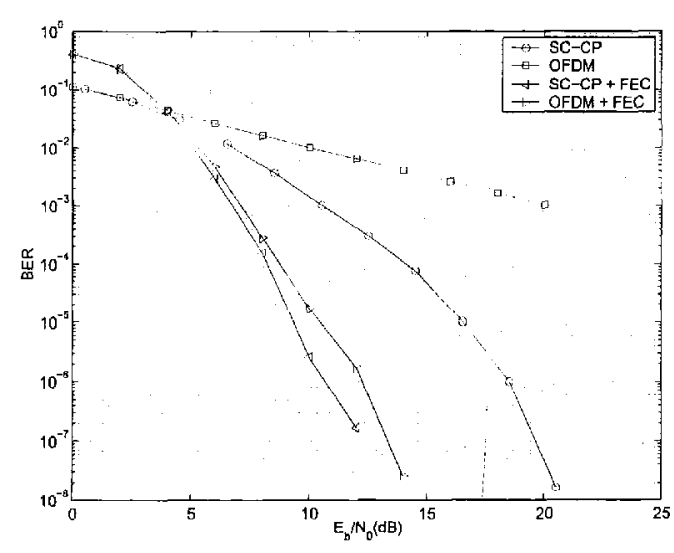

Figure 2: OFDM needs special attention to compensate for multipath fading

will show a high output value. On the other hand, when half of the subcarrier signals are in opposite phase with the other half, they will compensate each other and the output signal will have a low amplitude. Transmitting and receiving such signal requires an analog front-end with a high dynamic range and thus a low efficiency.

A third consequence involves frequency synchronization. At the receiver, the local oscillator must be synchronized within a fraction of the bandwidth. With OFDM however, this means a fraction of a subband, which is a factor $1 / N_{c}$ smaller than the signal bandwidth. This puts a higher constraint on the local oscillator circuitry of the receiver.

\section{Interference Models}

In the previous section, we have introduced two modulation techniques suitable for indoor communication. We have evaluated their performance in terms of BER over a multipath fading channel. However, a communication system also has to cope with different kinds of interference, mainly gen-

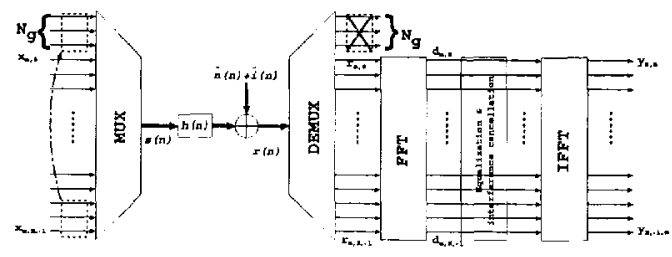

(b) SC-CP erated by other communication systems and/or other electronic equipment. The $2.4 \mathrm{GHz}$ ISM band in particular has to cope with interference generated by Bluetooth and microwave ovens. In this section, we will introduce the two baseband models we developed for Bluetooth and microwave oven interference.

\subsection{The Bluetooth Model}

The system described in this paper is not the only commercial communication system using the ISM-band. One particular system that is believed to become a very widespread $2.4 \mathrm{GHz}$ system in the near future, is Bluetooth[3]. In order to analyze our system in the presence of a Bluetoothinterferer, we have implemented a general model for such an interferer[4]. The Bluetooth-system makes use of FrequencyHopping Spread-Spectrum (FH-SS) to transmit data. The carriers are modulated with a Continuous-Phase Frequency Shift Keyed signal (CP-FSK). In the model, a random sequence of ASK-symbols represent the data and a time varying carrier $f_{c}(t)$ is used to model the frequency-hopping. The general form of the interferer is given by :

$$
\begin{gathered}
i_{F H}(t)=2 A \cos \left(2 \pi f_{c}(t) t+\pi m \int_{-\infty}^{t} d(\tau) d \tau\right) \\
\text { with } d(t)=\sum_{n=-\infty}^{+\infty} I_{n} \Pi_{T}(t-n T)
\end{gathered}
$$

where $I_{n}$ represents the data sequence and $\Pi(t)$ is a rectangular window.

$$
\Pi_{T}(t)= \begin{cases}1 & \text { if } 0<t<T \\ 0 & \text { otherwise }\end{cases}
$$

The modulation index of the CP-FSK signal of Equation (1) is given by :

$$
m=2 T \Delta f
$$

in which $T$ is the symbol-duration and $\Delta f$ the minimum frequency distance between two modulated symbols. 
The time-varying carrier frequency is expressed as follows :

$$
f_{c}(t)=\sum_{k=-\infty}^{k=+\infty} F_{k} \Pi_{T_{\text {bop }}}\left(t-k T_{h o p}\right)
$$

with $F_{k}$ the hopping-sequence.

We use this interference model with the parameters as listed in Table 1 to generate a Bluetooth interference signal. The Bluetooth-system is characterized by a data-rate of up to $1 \mathrm{Mbps}$. The instantaneous bandwidth is $1 \mathrm{MHz}$. This signal is hopping over 79 different hopping frequencies at a rate of 1600 hops/s. The different hopping frequencies are spaced apart by $1 \mathrm{MHz}$ and range from $2.402 \mathrm{GHz}$ to $2.48 \mathrm{GHz}$. Figure 3 depicts the spectrum of a Bluetooth system as it is computed by our model.

\begin{tabular}{|l|l|}
\hline$I_{n}$ & Random BPSK-sequence, $I_{n} \in\{-1,+1\}$ \\
$\mathrm{T}$ & $1 \mu s$ \\
$\mathrm{~m}$ & 0.3 \\
$T_{h o p}$ & $625 \mu s$ \\
$F_{k}$ & $\{2.402 \mathrm{GHz}, 2.403 \mathrm{GHz}, \ldots, 2.48 \mathrm{GHz}\}$ randomly \\
& shuffled and periodically extended \\
$f_{B B}$ & $2.44 \mathrm{GHz}$ \\
$\mathrm{A}$ & $1 / \sqrt{2}$ \\
\hline
\end{tabular}

Table 1: The Bluetooth-system as a special case of the general interference model

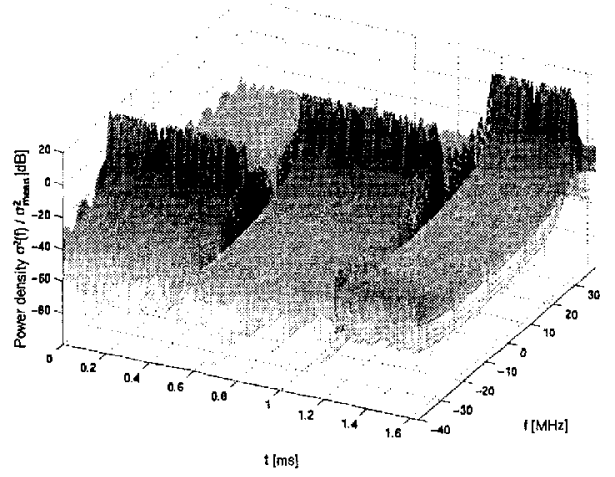

Figure 3: A Bluetooth-interference hops through the ISMband

\subsection{The Microwave Oven Emission Model}

Based on the physical properties of a microwave oven[5], a model for the interference of this device was derived. The spectral properties of this signal are depicted in Figure 4. In this figure, the spectrum is shifted over the design-frequency of the microwave oven. Two different phases are present : during one half of the period of the line-voltage the interference is absent; during the other half the interference is present. At the beginning of the on-phase, the microwave oven generates a broadband signal which soon transforms in a relative narrowband signal with a central frequency under $2.45 \mathrm{GHz}$. Progressively, this signal becomes narrower in bandwidth and the central frequency evolves towards $2.45 \mathrm{GHz}$, which is the design frequency of a microwave oven. Once the design frequency is reached, the signal remains stable at this frequency with a bandwidth of $1 \mathrm{MHz}$. At the end of the on-phase, the interference signal traverses the same traject in the opposite direction.

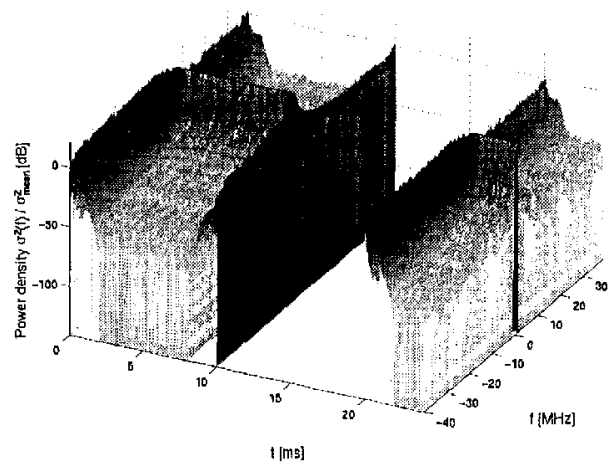

Figure 4: One cycle of a microwave oven spectrum

\section{Interference Mitigation Strategies}

An indoor communication system suffering from interference will show a largely degraded performance. In case of narrowband interference, the energy of the interfering signal drowns the energy of the desired signal in a part of the signal bandwidth. The influence this has on a communication system depends on the type of modulation used and interference mitigation strategy applied. This section introduces three interference mitigating strategies for the two modulation techniques introduced in section 2.

\subsection{OFDM}

Narrowband interference, added to a multicarrier signal such as OFDM, will corrupt the information transmitted on the affected subcarriers. Every subcarrier transmits on the average $1 / N_{c}$-th of the total transmitted energy, with $N_{c}$ the number of subcarriers. If the interference energy is concentrated on a few of those subcarriers, it drowns the useful signal and thereby destroys the information on those carriers. This results in a flooring-effect of the BER vs. $E_{b} / N_{0}$ since the transmission errors are no longer caused by the noise, but rather by the interference (Figure 5). 


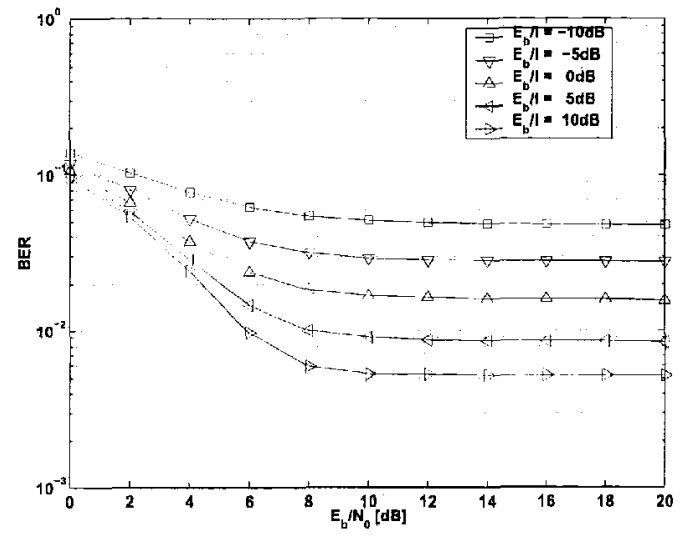

Figure 5: Interference causes a floor of the BER performance

We propose three interference mitigation strategies that will also avoid or compensate for multipath fading effects, which are similar to the effects of interference in the sense that they also destroy the information on a number of subcarriers.

\subsubsection{Adaptive Loading}

Due to multipath fading and interference, there is a large difference in transmit capacity between the OFDM subcarriers. The overall system performance is limited by the worst performing subcarrier. To avoid this limitation, we can optimize the transmitted information on each subcarrier towards the information capacity of that subcarrier. This capacity is determined by the noise and interference power received on the subcarrier. The technique which implements this optimization is called adaptive loading. It has already successfully been used in the Asynchronous Digital Subscriber Line (ADSL) technology and is gaining interest in the field of Wireless Local Area Networks (WLAN) [6]. We are performing extensive research on this technique and are considering it also for interference mitigation. Figure 6 shows the principle of adaptive loading. Subfigure (a) shows a typical frequency response of an indoor channel with a fading dip of $30 \mathrm{~dB}$. Subfigure (b) shows an example of the power distribution of a narrowband interferer with a peak-power of $14 \mathrm{~dB}$ above the noise level. Subfigure (c) and (d) respectively show the rate distribution and the power distribution over the different subcarriers. It is clearly shown that the most affected subcarriers are not carrying any data. Subfigure (e) shows how the Signal to Noise and Interference Ratio (SINR) is almost equally spread over the available subcarriers. In a non-adaptive transmission system, the rate and the transmit power would equally be distributed over all available subcarriers. Since nature does not equally spread noise and interference, this results in large SINR differences between the subcarriers. Finally we can see in subfigure (f) how the BER is flattened over the different useful subcarriers. Chow, Cioffi and Bingham have proposed an algorithm yielding the optimal distribution of transmit power and transmission rates over the available subcarriers[7]. The optimal transmission rate for eyery subcarrier, $R_{k}$, is directly determined by Equation (4).

$$
R_{k}=l d\left(1+\frac{S_{k}}{N_{k} \Gamma}\right)
$$

$\Gamma$ is an iteratively determined parameter which makes $\sum_{k} R_{k}=R_{t o t}$ with $R_{t o t}$ the predefined number of information bits assigned to one OFDM-symbol. J. Fisher and J. Huber proposed a bit loading algorithm based on this strategy[8]. In this algorithm, the carrier rates are iteratively computed until the desired rate $R_{\text {fot }}$ is reached. The algorithm takes the following parameters as an input : the subcarrier channel response $h_{k}$, the subcarrier noise plus interference power $N I_{k}$, the total number of bits per OFDM-symbol $R_{t o t}$ and the total transmit power $S_{t o t}$.

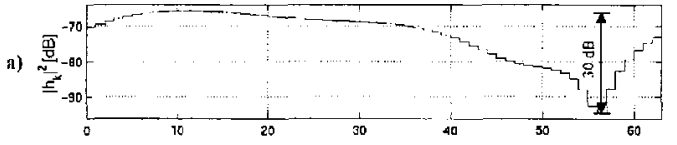

b)

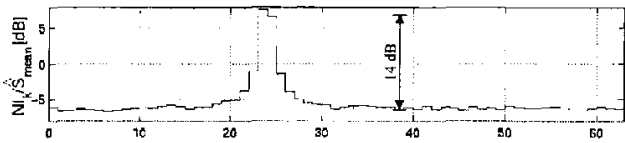

c)

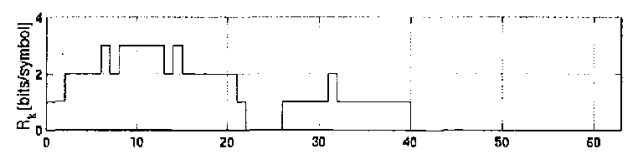

d)

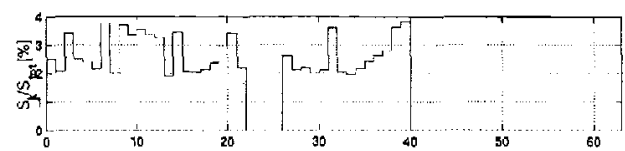

c)
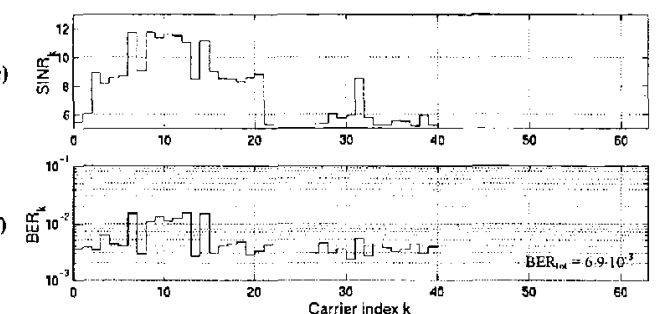

Figure 6: Adaptive loading optimizes the overall system performance

The adaptive loading scheme requires that both $h_{k}$ and $N I_{k}$ 
are measured at the receiver and fed back to the transmitter. In our simulations, this is done during a silent phase with a duration of 10 OFDM-symbols. Since $h_{k}$ and $N I_{k}$ cannot be updated during transmission, the adaptive loading system assumes a quasi-static channel response and interferer. This immediately indicates one of the major drawbacks of the system, i.e. the technique cannot track dynamic interference sufficiently.

\subsubsection{Spreading}

One of the drawbacks of the adaptive loading scheme is that channel and interference update information must be fed back periodically from the receiver to the transmitter. Therefore, this system will only work properly in a quasi-static environment. In the case of a Bluetooth hopping interferer, a complete transmitted block can be lost when the Bluetooth signal hops just after the update-phase of the parameters. Another drawback is that adaptive loading only works for point-topoint connections and does not support broadcasting. Therefore we implemented a second interference mitigation strategy which does not have these drawbacks. This technique exploits frequency diversity by applying Direct Sequence Spreading (DSS) in the frequency-domain. This technique is based on a strategy proposed by K. Fazel [9] which we have improved. The idea is to transmit the same data over multiple carriers in such way that the frequency separation is maximized. At the receiver, both copies of the data are then recombined in an optimal way, as is shown in Figure 7. Whenever narrowband interference or multipath fading affects one subcarrier, this data can still be retrieved by using its copy from another subcarrier. The receiver performs the despreading by using an estimate of the SINR of the received signal. This estimate is used to compute the weighting-factor by which the signal of each subcarrier must be rescaled such that the recombined signal results in a maximized detection probability. We can write the estimate of the received bit as follows :

$$
\begin{aligned}
\hat{x} & =\sum_{k=1}^{L} w_{k}\left(h_{k} c_{k} x+n i_{k}\right) \\
& =x \sum_{n=1}^{L} w_{k} h_{k} c_{k}+\sum_{k=1}^{L} w_{k} n i_{k}
\end{aligned}
$$

in which $w_{k}$ represent the weighting factor, $h_{k}$ the appropriate channel frequency response and $c_{k}$ the spreading code. $n i_{k}$ Represents the noise plus interference on the $k^{\text {th }}$ subcarrier. The optimal weighting factors are computed with Equation (6):

$$
w_{k}=\frac{h_{k}^{*} c_{k}^{*} / N I_{k}}{\sum_{l=1}^{L}\left|h_{l}\right|^{2} / N I_{l}}
$$

This maximum-ratio combining requires an estimate of both the channel coefficients and the average noise-and-interference power (NI) on the subcarriers. The channel coefficients can be reused from the equalization unit. The original implementation from K. Fazel used zero-pilots to compute an estimate for NI. These zero-pilots reduce the bandwidthefficiency of the system. To avoid this, we have replaced these zero-pilots by an estimator based on decision feedback. This estimator maintains the system performance and does not reduce the systems bandwidth efficiency.

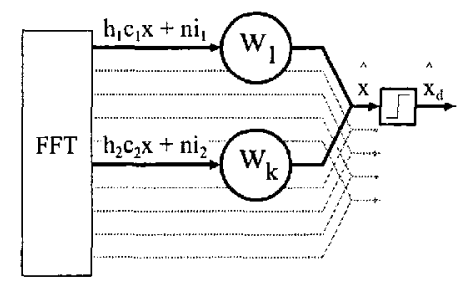

Figure 7: Optimal combining increases the system performance

\subsection{SC-CP}

In the previous section, we have introduced two interference mitigation strategies for an OFDM-transmission system. The multi-carrier property of OFDM enables us to apply an interference-mitigating strategy independently for every subcarrier and thus for every transmitted data-symbol. This property is the biggest difference between interference mitigation in an OFDM-system and in a SC-CP-system. In the latter case, the energy of every transmitted bit is spread over the complete signal bandwidth. Every impairment within this bandwidth will thus have its impact on every transmitted symbol. Since it is not possible to avoid the transmission of information in a certain part of the bandwidth, the mitigation strategy is aiming at ignoring the affected frequencies and reconstructing the corrupted signal.

\subsubsection{FRESH-filtering}

The interference mitigation strategy we propose for the SCCP modulation technique is based on a technique introduced by W.A. Gardner[10]. This technique uses the spectral correlation property of a signal transmitted with an excess bandwidth. This excess bandwidth introduces frequency diversity by transmitting one or more spectral images of the signal. A correlation exists between the signal and a fixed predefined frequency-shifted copy of the signal or its complex conjugate. The frequency-shifts over which this correlation occurs depend on the type of modulation used, but is always a linear combination of the baudrate, the carrier frequency or both. In case of QPSK-modulation or higher QAM-constellations, correlation occurs only for linear combinations over the baudrate. A list of frequency correlations is published in[10]. We can now use the frequency correlation property of the signal to correct the distortion generated by the interference. This principle is depicted in Figure 8. First we have to gen- 


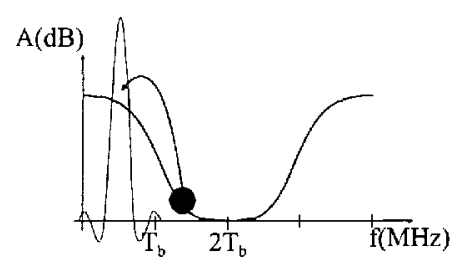

Figure 8: Frequency correlation can be used to correct the corrupted signal

erate all the frequency-shifted versions of the input-signal. Then we apply a Linear Time-Invariant filtering-operation (LTI) on all these signals and finally we combine all the results to generate the corrected signal. This complete operation is called FRequency SHifted filtering (FRESH-filtering). The filters used are computed in such a way that the overall performance is optimized. We can write the signal after FRESH-filtering as follows[11]:

$$
\hat{s}(t)=\mathbf{w}^{H} \mathbf{y}(t)
$$

Here, w corresponds to the Least Square (LS) coefficients of the LTI-filters minimizing the average-squared error, \langle|$\hat{s}(t)-$ $\left.\left.s(t)\right|^{2}\right\rangle$. The transmitted data-sequence is $s(t)$ and $\hat{s}(t)$ is the estimate of the transmitted data-sequence at the receiver. The definition of $y$ is as follows :

$$
\begin{aligned}
\mathbf{y}(t) \triangleq & {\left[p_{\alpha_{0}}\left(t-t_{1}\right) \cdots p_{\alpha_{0}}\left(t-t_{m}\right) \cdots\right.} \\
& p_{\alpha_{L-1}}\left(t-t_{1}\right) \cdots p_{\alpha_{L-1}}\left(t-t_{m}\right) \\
& p_{-\beta_{0}}\left(t-t_{1}\right)^{*} \cdots p_{-\beta_{0}}\left(t-t_{m}\right)^{*} \\
& \left.p_{-\beta_{M-1}}\left(t-t_{1}\right)^{*} \cdots p_{-\beta_{M-1}}\left(t-t_{m}\right)^{*}\right]^{T}
\end{aligned}
$$

in which $p_{\mu} \triangleq r(t) e^{i 2 \pi \mu t}$ which is a frequency-shifted version of the received signal $r(t), t_{k}$ are delays chosen such that the designed filters are causal. The LS-coefficients $\mathbf{w}$ are now computed as follows :

$$
\begin{aligned}
\mathbf{w}= & \hat{R}_{y y}^{-1} \hat{R}_{y s} \\
& \hat{R}_{y y}=\left\langle y(t) y^{H}(t)\right\rangle_{N} \\
& \hat{R}_{y s}=\left\langle y(t) s^{*}(t)\right\rangle_{N}
\end{aligned}
$$

with $\langle.\rangle_{N}$ a time averaging over $\mathrm{N}$ samples and $y^{H}(t)$ denotes the complex conjugate transposition.

We have implemented a system with an excess bandwidth of $100 \%$ and 16-QAM as modulation technique. This means that we have a correlation available between three different frequency-shifts, namely $r(t), r(t) e^{i 2 \pi f_{1} t}$ and $r(t) e^{-i 2 \pi f_{1} t}$ with $f_{1}$ the baudrate of the signal. The data-stream is subdivided into blocks of 8000 bits and a training sequence is transmitted before every block. This sequence is used to initialize the filter coefficients. The FRESH-filter coefficients are computed in the time-domain using Equation (9) and afterwards transformed to the frequency-domain using the FFT-algorithm.

The LS-coefficients computed with Equation (9) will minimize the mean-squared error between the received signal and the training signal. This error can either be caused by interference or by multipath fading. FRESH-filtering will not only compensate for interference, but also for the channelimpairments. There is thus no need for an extra equalization step.

In order to successfully use FRESH-filtering for interferencecancellation, we have to avoid spectral leakage of the interfering signal. At the transmitter, we have constructed a datasignal which is pseudo-cyclic. This property is important because it reduces all filtering operations in the receiver to a complex multiplication in the frequency-domain. A problem occurs however when an interference-signal is added to the data-signal. This interfering signal is not cyclic. An FFT-transformation from a non-cyclic signal will result in a spectral leakage[2]. This leakage will result in an increased signal distortion. The undesired spectral interference sidelobes are not yet present when the FRESH-coefficients are computed. The extra impairment will thus not be removed by the FRESH-filters and the system performance will decrease. Since the impairment is caused by the non-cyclic behaviour of the interference, it will have its biggest impact at the borders of the block-symbols. To avoid extra transmission errors, we have introduced a small silent-phase at the beginning and end of every block-symbol. This is equivalent to the zero-carriers which are introduced in an OFDMsymbol to avoid spectral out-of-band leakage.

\section{3 performance comparison of OFDM and SC-CP dis- torted by interference}

This subsection presents the performance of both simulation techniques when affected by the narrowband interference as described in section 3.1 and section 3.2. The transmission rate of both systems was fixed at $20 \mathrm{Mbps}$ with a signal bandwidth of $10 \mathrm{MHz}$. The simulations arc performed for an OFDM-system using both spreading and adaptive loading as interference mitigation strategy and SC-CP with FRESH-filtering. As a reference, we also performed all simulations for both systems without interference mitigation. For a fair comparison, we kept the bandwidth efficiency constant. Both OFDM with spreading and SC-CP with FRESH-filtering are thus using 16-QAM constellations to compensate for the extra required bandwidth for the frequency spreading. The reference simulations use QPSK constellations. Both the adaptive loading scheme for OFDM as the FRESH-filter scheme for SC-CP use a training period to initialize the interference mitigation parameters. This training-period is inserted periodically. The length of the 
data-blocks transmitted between subsequent training periods is fixed at 8000 databits.

On Figures 9 and 10, the striped lines represent the performance of the reference system, while the solid lines represent the performance of the different systems using interference mitigation. Figure 9 shows the performance of the different systems suffering from a static narrowband interferer. Figure 10 shows the performance of the same systems affected by a dynamic microwave oven interferer.

The flooring-effect caused by the interferer is more pronounced in case of OFDM-modulation, where the interference completely destroys the information on certain subcarriers. In case of static narrowband interference, the influence of interference is successfully removed, as the flooring-effect has disappeared. When no interference is present ( $100 \mathrm{~dB}$ SIR), the interference mitigation strategies induce a performance loss caused by the increased constellation size, which we introduced to keep the bandwith efficiency constant.

Figure 10 shows the performance of both transmission systems suffering from a dynamic microwave interferer. Both adaptive loading and FRESH-filtering are less effective against dynamic interference as they require a periodic training-phase in which the interference mitigation algorithm is initialized. However, whenever the characteristics of the interference changes, the initialization is no longer valid. This will happen whenever the microwave oven switches on or off. In the worst case, the interference characteristics will change shortly after the initialization phase, such that the entire datablock is lost. In case of microwave-interference, this effect is amplified by the broadband interference during on- and offswitching of the magnetron element. During those phases, the microwave will completely corrupt the signal bandwidth, such that all data is lost.

Simulations have also been performed with the two modulation techniques suffering from a frequency-hopping Bluetooth interferer. These simulations result to similar conclusions. Both OFDM with adaptive loading and SC-CP with FRESH-filtering show a residual performance floor due to the mismatch between the actual interference signal and the periodical initialization of the interference mitigation algorithm. OFDM with DSS performs better under these circumstances, since the SINR estimating feedback loop is able to track the hopping interference. This way, DSS can avoid the loss of a complete transmission block.

\section{Conclusions}

In this paper, OFDM and SC-CP are presented as two different modulation techniques suitable for in-house wireless digital communication. Interference mitigation was performed by applying direct-sequence spreading and adaptive loading for OFDM-modulation and FRESH-filtering for SC-CPmodulation in the presence of a multipath channel and interference. The interference mitigation strategies were simulated on a Gaussian channel suffering from two distinct types of interference : a static narrowband interference and a microwave oven interference. The simulations on static interference show that we can successfully mitigate this interference for both modulation techniques. The performance results for a microwave oven interference show that the influence of this interferer can be reduced, but a residual error floor still remains. This is caused by the periodic broadband interference generated by the microwave oven. This kind of interference cannot be completely mitigated.

Similar simulations on a Biuetooth interferer show that in this case the DSS strategy is the most effective. Since both adaptive loading and FRESH-filtering rely on the periodic system initialization during a silent phase, they cannot track a dynamic Bluetooth interferer as effectively as the DSS algorithm. From these results, we can conclude that the proposed algorithms must be made adaptive.

On a digital processing level, the OFDM-technique requires less complexity. However the properties of the OFDM-signal put a high constraint on the analog front-end required. Therefore, a meaningful trade-off between both systems must be made on a higher system-level, taking all the analog and digital effects into account. 


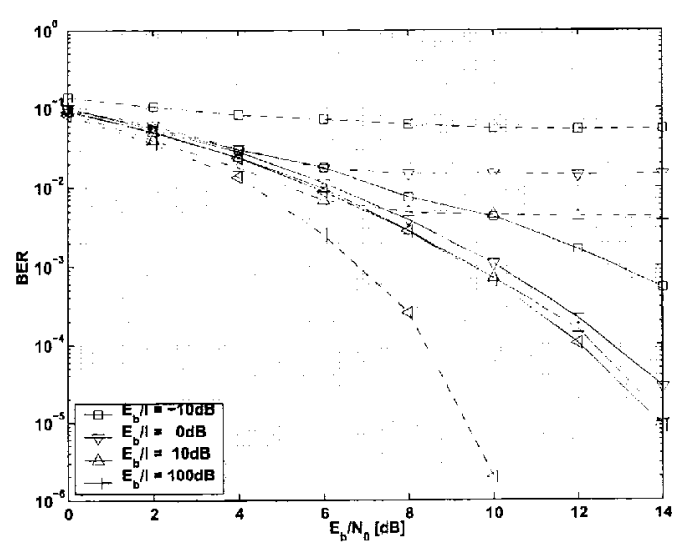

(a) OFDM with adaptive loading

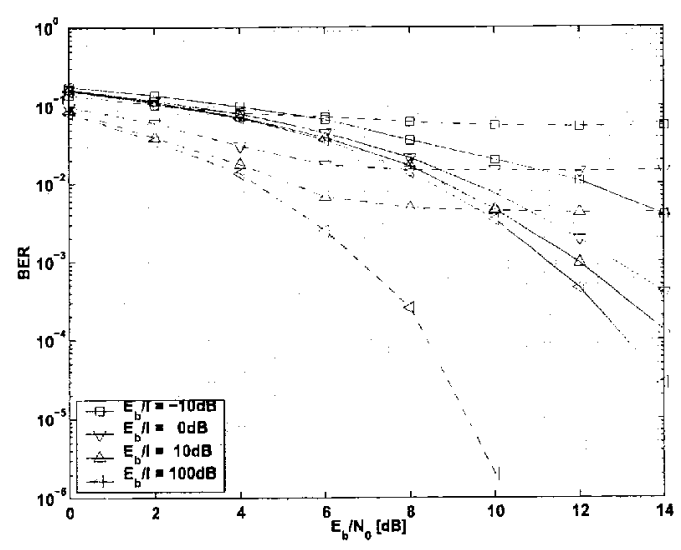

(b) OFDM with direct sequence spreading

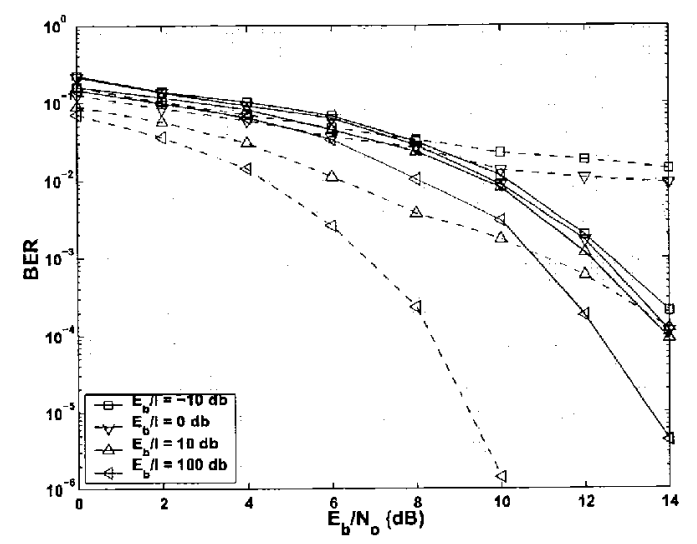

(c) SC-CP with FRESH-filtering

Figure 9: Static interference is successfully removed

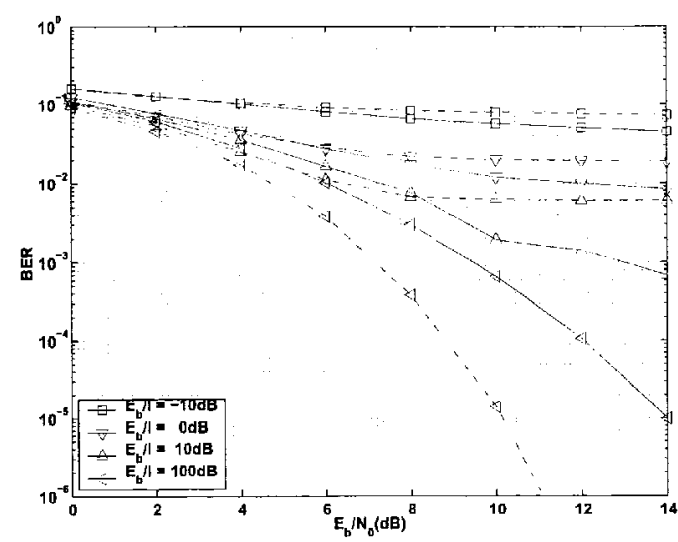

(a) OFDM with adaptive loading

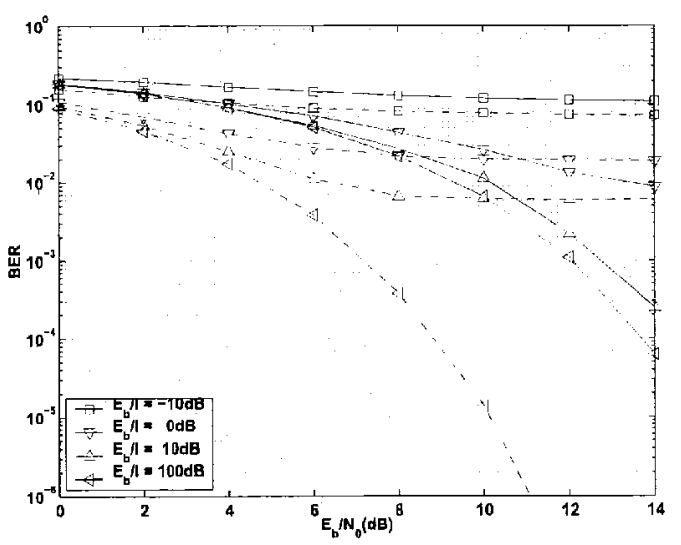

(b) OFDM with direct sequence spreading

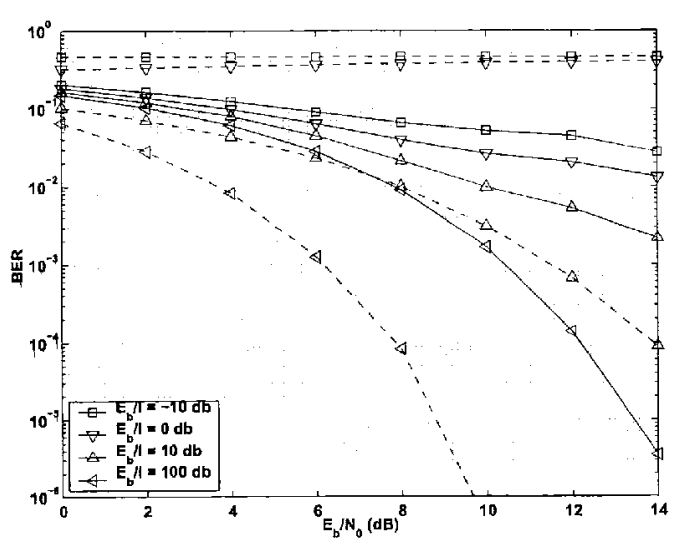

(c) SC-CP with FRESH-filtering

Figure 10: Dynamic interference of a microwave oven is less effectively mitigated 


\section{Acknowledgements}

The authors wish to thank P. Vandenameele, W. Eberle and $S$. Thoen for the fruitful discussions. This work was funded by the IWT.

\section{References}

[1] P. Vandenameele, L. Van der Perre, B. Gyselinckx, M. Engels, and H. De Man, An SDMA algorithm for high-specd WLAN. Performance and Complexity. In IEEE Globecom 1998 Communications, volume 1, pages 189-194, November 1998.

[2] J.G. Proakis and D.G. Manolakis. Digital Signal Processing, principles, algorithms and applications. Prentice Hall, 1996.

[3] The bluetooth and homerf homepages. http://www.bluetooth.com http://www.homerf.org.

[4] R. Ness. Design of an OFDM Transmitter/Receiver. Master's thesis, Universität Karlsruhe (TH). Ecole Nationale Supćricure d'Elcctroniquc et de Radioćlectricité de Grenoble, 1999.

[5] L. Leyten and H. Visser. Radiation propertics of the microwave oven. Technical report, Nat. Lab., Philips Electronics, 1993.

[6] L. Van Der Perre, S. Thoen, P. Vandenameele, B. Gyselinckx, and M. Engels. Adaptive loading strategy for a high speed OFDM-based WLAN. In IEEE Globecom 1998 Communications, volume 4, pages 1936-40, 1998.

I7] P.S. Chow, J.M. Cioff, and J.A.C. Bingham. A practical discrete multitone transceiver loading algorithm for data transmission over spectrally shaped channels. IEEE Transactions on Communications, 43:773-775, April 1995.

[8] R.F.H. Fisher and J.B. Huber. A new loading algorithm for discrete multitonc transmission. In IEEE Globecom 1996 Communications, volume 1, pages 724-8, November 1996.

[9] K. Fazcl. Narrowband Interference Rejection in Orthogonal Multjcarricr Sprcad-Spectrum. In IEEE Proc., third annual international conference on universal personal communications, pages Xvii +674 , 1994.

[10] W. A. Gardncr. Cyclic Wiener Filtering: Theory and Method. IEEE Trans. on Comm., 41, no. 1:151-163, January 1993.

[11] W.A. Gardncr, G.K. Ycung, and W.A. Brown. Signal reconstruction after severe spectral excision. In Conference Record of the twentyninth Asilomar Conference on Signals, Systems and Computers, volume 1, pages 516-20. IEEE Comput. Soc. Press, 1996.

\section{Biographies}

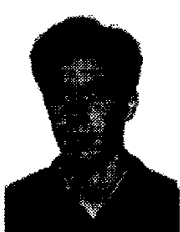

Geert Carron was born in Belgium in 1973. He received his ing. degrec in Elcctrical Enginecring from the K.I.H. De Nayer, Belgium in 1995 and his M.S. degree in Elcctrical Engineering from the K.U.Leuven, Belgium, in 1997. Currently, he works as a rescarcher in the Wireless Systcms group of IMEC, were he is working at wireless highspeed in-house networks.

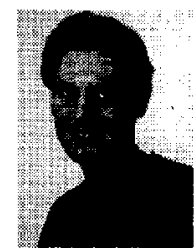

Rcto Ness received the engineering degree in Electrical Engineering from the University of Karlsruhe, Germany and from the Ecole Nationale Superieure d'Electronique et de Radioélectricité de Grenoble, France, in 1999. He carricd out his thesis in the Wireless Systems group at IMEC, Belgium, where he focussed on narrow-band interference cancellation in OFDM-based WLANs. Currently, he works in the development department of Tenovis GmbH \& Co. KG, Germany.

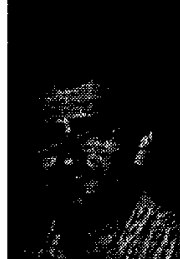

Luc Deneire (M'99) was born in Belgium in 1964. He received the Eng. degree in Electronics from University of Licge (Belgium) in 1988, the Eng. degree in Telecommunications from University of Louvain-La-Neuve in 1994 and the $\mathrm{PhD}$ degrec in Signal Processing at Eurecom, Sophia-Antipolis, France in 1998. In 1999, he was consultant for Texas Instruments, Villencuve-Loubet, France and since late 1999, he is a senior researcher at IMEC. He is working on the signal processing algorithms involved in wireless communications, specifically for Wireless LANs and LEO Satellite Systems. His main interests are equalization and chamel cstimation, smart antennas and link adaptation.

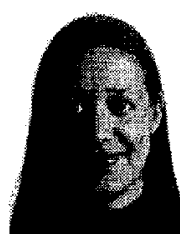

Liesbet Van der Perre received the M.S. degree in Electrical Enginecring from the K.U.Leuven, Belgium, in 1992. She performed her M.S. thesis research at the ENST in Paris, France. She received the Ph.D. degree in Electrical eniginecring from the K.U.Leuven in 1997. Currently, she works as a senior researcher in the Wireless Systems group at IMEC. Her work focuses on system design and digital modems for high speed wireless communications. Also, she is a part-time professor at the University of Antwerp, Belgium.

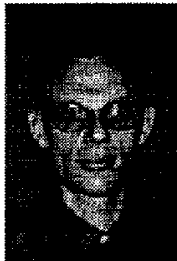

Marc Engels is the director of the telecom departement (DISTA) at IMEC. His main research activity is in the implementation of telecommunication systems on a chip. His current work is focussed on wireless systems, such as WLAN and satellite communication. For these systems, the department investigates the DSP processing, the mixed-signal RF front-end and run-time configurable softwarc. A major cmphasis of the department is also on a $\mathrm{C}++$-based design methodology to realise these applications onto VLSI in an cfficient way.

Previously, he performed research at the Katholieke Universiteit Leuven, Belgium; Stanford University, CA, USA; and the Royal Military School, Brussels, Belgium. Marc Engels received the engineering degree (1988) and the Ph.D. (1993), both from the Katholieke Universiteit Leuven, Belgium. Marc Engels is an active member of the SITEL, the KVIV telecommunications society and the IEEE Benelux chapters on signal processing and telecommunications. He is also an associated editor of trans. on VLSI. 